\title{
EVALUACIÓN TERMODINÁMICA DE VARIABLES CRÍTICAS EN LA ESTABILIDAD DE LA PANELA DE CAÑA DE AZÚCAR
}

\author{
THERMODYNAMIC EVALUATION OF CRITICAL VARIABLES IN THE STABILITY OF PANELA \\ OF SUGAR CANE
}

\section{Lina Constanza BOLAÑOS-CARDONA', Ingrid Alejandra BRIÑEZ-JAVELA', Juan Sebastián RAMÍREZ-NAVAS ${ }^{1,2}$ *}

1 Programa de Química, Grupo Giema, Universidad Santiago de Cali, Calle 5 \# 62-00, Campus Pampalinda, Cali, Colombia.

2 Escuela de Ingeniería de Alimentos, Grupo Giema, Universidad del Valle, Sede Meléndez, Cali, Colombia.

* Autor corresponsal. E-mail: juan.ramirezo6@usc.edu.co
Historia del artículo

Recibido: Noviembre 11, 2017

Evaluado: Febrero 5, 2018

Aceptado: Abril 13, 2018

Disponible: Mayo 3, 2018

\section{Resumen}

El objetivo de este trabajo es determinar la vida útil de la panela mediante la evaluación de variables críticas de estabilidad. Para tal fin, se almacenaron tres presentaciones comerciales de panela (granulada, cuadrada y redonda) durante 129 días en cámaras bioclimáticas de dos ambientes de almacenamiento (ambiente: $30 \pm 2{ }^{\circ} \mathrm{C} / 75 \pm 5 \% \mathrm{HR}$; y acelerado: $40 \pm 2{ }^{\circ} \mathrm{C} / 75 \pm 5 \% \mathrm{HR}$ ). Se evaluaron los parámetros de humedad, azúcares totales y actividad acuosa en las muestras previamente homogenizadas a los tiempos 0, 27, 35, 55, 76 y 129 días. Debido al incremento de la actividad acuosa y la ganancia de humedad se evidenció cambio en la calidad del producto, variación del color y la textura, con señales de reblandecimiento (panela cuadrada y redonda) y aglutinación (panela granulada). Con los resultados obtenidos se predice que, al almacenar a $25^{\circ} \mathrm{C}$, los tiempos de vida útil para las muestras son: 398 días para panela redonda, 164 días para panela cuadrada, y 206 días para panela granulada.

Palabras clave: panela; caña de azúcar; actividad de agua; vida útil; azúcares totales; humedad

\section{Abstract}

The objective of this work was to determine the shelf life of panela by evaluating critical stability variables. For this purpose, three commercial presentations of panela (granulated, square and round) were stored for 129 days in bioclimatic chambers of storage environments (ambient: $30 \pm 2{ }^{\circ} \mathrm{C} / 75 \pm 5 \% \mathrm{RH}$ and accelerated: $40 \pm 2{ }^{\circ} \mathrm{C} / 75 \pm 5 \% \mathrm{RH}$ ). The parameters of moisture, total sugars and water activity were evaluated in samples previously homogenized at times 0, 27, 35, 55, 76 and 129 days. Due to the increase in water activity and moisture gain, there is a change in product quality, variation of the color and texture, with signs of softening (square and round panela) and agglutination (granulated panela). With the results obtained, it is predicted that by storing at $25^{\circ} \mathrm{C}$ the useful life times for the samples are: 398 days for round panela 164 days for square panela and 206 days for granulated panela.

Keywords: panela; sugar cane; water activity; shelf life; total sugar; moisture

\section{INTRODUCCIÓN}

La panela es un edulcorante natural que se obtiene por la concentración de los jugos de la caña de azúcar y la posterior cristalización de la sacarosa. Esta no se somete a ningún proceso de refinado, centrifugado y depuración. Se caracteriza por su alta concentración de azúcares, contenido de minera- 
les y trazas de vitaminas provenientes de la caña de azúcar que se conservan en la panela (Álvarez Londoño, 2005). Es posible encontrar diferentes presentaciones comerciales de panelas (Acurio, 2010). Entre estas se encuentran panela cuadrada, rectangular, redonda, con cresta redonda y triangular, pulverizada o granulada, entre otras.

A pesar de la importancia de la panela, la agroindustria ha sufrido una serie de problemas relacionados con la baja productividad, la deficiencia de la calidad del producto, la organización de socios, los problemas de mercado y los impactos sobre el ambiente (Sandoval et al., 2007). Adicionalmente, el tiempo de vida útil de las presentaciones comerciales de panelas se ha visto afectado de forma negativa por problemas técnicos, tales como la variación de la actividad acuosa, la humedad y los azúcares invertidos (Acurio, 2010).

La panela, al ser un producto higroscópico, presenta signos de deterioro por contaminación microbiana; este efecto se debe al nivel tan alto de actividad acuosa. La concentración de azúcares y el nivel del agua en la panela pueden variar de acuerdo con el genotipo, la época de cosecha y el tipo de manejo de extracción y posextracción del jugo de la caña, de la concentración de la miel y el batido de la panela (Uppal et al., 2005). Es importante conocer los parámetros químicos y fisicoquímicos (tanto termodinámicos como cinéticos) de la panela; por ejemplo, el contenido de humedad, los azúcares invertidos, la actividad de agua, el coeficiente de actividad, la entalpía molar parcial y el tiempo de vida útil (Alvarado, 2012; Badui Dergal, 2006).

Entre los factores que intervienen en el deterioro de los alimentos se encuentra la temperatura, la humedad, la luz y el aire, especialmente el oxígeno; sumado a estos factores se encuentra el tiempo (Vanaclocha y Requena, 2003). Para el caso de la panela, entre los factores más críticos y que causan mayor deterioro se encuentran la temperatura y la humedad tanto del producto como del ambiente.

El objetivo de esta investigación es analizar los cambios de la actividad acuosa durante el almacenamiento de muestras de panelas comerciales en diferentes presentaciones, con relación a la humedad y los azúcares. A partir de la información que arrojó el estudio se obtuvieron diversos parámetros termodinámicos y cinéticos que permiten controlar y predecir la estabilidad del producto durante el tiempo de almacenamiento.

\section{MATERIALES Y MÉTODOS}

El almacenamiento en cámaras climáticas y los ensayos fisicoquímicos para el estudio de las panelas se realizaron en el Laboratorio Fisicoquímico de Ángel Diagnostica de Colombia, por un periodo de cuatro meses. Se contó con disposición de equipos y reactivos por parte del laboratorio en mención.

\section{Panela}

Se trabajó con tres presentaciones de panela: granulada $(\mathrm{PG})$, cuadrada (PC) y redonda (PR), de la misma marca comercial. Se garantizó que cada presentación proviniera del mismo lote de fabricación (PG: TPNA/4 00:22 FF 22/02/2017; PC: TPNA/01 05:28 FF 07/02/2017; y PR: TPNA/03 20:11 FF 07/02/2017).

\section{Muestreo y almacenamiento}

Las muestras se almacenaron por un periodo de tres meses (129 días) en cámaras bioclimáticas, en dos ambientes de almacenamiento: 1 ) ambiente $30 \pm 2{ }^{\circ} \mathrm{C} / 75 \pm 5 \%$ humedad relativa en una cámara VM controles (modelo HR 700, Colombia), con un datalogger Brixco (modelo DT-172, Colombia); y 2) acelerada $40 \pm 2^{\circ} \mathrm{C} / 75 \pm 5 \%$ humedad relativa en una cámara control superior (modelo CS-CC720, Colombia) con un datalogger Tinytag (modelo Ultra 2, Inglaterra), ya que son las condiciones de estudio con frecuencia trabajadas en la literatura (Acurio, 2010).

La distribución de las muestras en las cámaras bioclimáticas se realizó de forma organizada en parrillas diferentes. Cada presentación se ubicó en un nivel de parrilla, de modo que se evitó la contaminación cruzada. Además, las muestras se almacenaron en su respectiva bolsa o empaque original bien sellado.

Antes del análisis fisicoquímico las muestras se homogeneizaron y luego se realizó un cuarteo con el fin de obtener una muestra representativa para los análisis. En este, la panela redonda y la cuadrada se rallaron y la granulada solo se cuarteó.

\section{Parámetros fisicoquímicos}

Las determinaciones de los parámetros de humedad (H), actividad acuosa $\left(a_{w}\right)$ y contenido de azúcares totales (AT) se realizó en las muestras previamente homogeneizadas a los tiempos 0, 27, 35, 55, 76 y 129 días, en las tres presentaciones (PC, PC y PR). Los análisis de humedad y el contenido de azúcares totales se realizó según la Resolución 779 de 2006 (MinSalud, 2006), y la Norma Técnica Colombiana NTC 1311 Productos Agrícolas. Panela (Icontec, 2009). Véase la (Tabla 1).

\section{Actividad de agua}

La determinación de la actividad acuosa se realizó con un medidor digital Decagon (modelo PawKit, EE.UU.). Se colocó aproximadamente $1 \mathrm{~g}$ de muestra de panela previamente homogeneizada en la cámara portamuestra, luego se oprimió el botón I, de manera que el equipo realizó la lectura y, al cabo de aproximadamente cinco minutos, arrojó el valor de $a_{w}$. Posterior a esto, se volvió oprimir el botón I para seguir con la otra lectura. La verificación del medidor se realizó con un estándar de cloruro de sodio de concentración $6 \mathrm{~mol} / \mathrm{kg}$ de $0.76 \mathrm{a}_{\mathrm{w}}$ (Aqualab, EE.UU., lote 1688045).

\section{Humedad}

La determinación del \%H se realizó de acuerdo con la AOAC 925.45 (AOAC, 2005). La determinación de humedad por medio de una estufa de secado a temperatura $105^{\circ} \mathrm{C} \pm 2^{\circ} \mathrm{C}$. Se pesó en una balanza analítica Ohaus (modelo PA214C, EE.UU.), aproximadamente $5 \mathrm{~g}$ de muestra homogeneizada en cajas metálicas con tapa, luego se llevó al horno de convección forzada Binder (modelo FD 115-UL, Alemania) por $3 \mathrm{~h} \mathrm{a} 105^{\circ} \mathrm{C}$. Posteriormente, se llevó al desecador hasta que alcanzó la temperatura ambiente y se pesó. Se introdujo a la estufa por 1 h, se llevó al desecador hasta $T_{\text {amb }}$ y se pesó. Se repitió este proceso hasta que la diferencia entre los pesos finales fuera menor de $5 \mathrm{mg}$. Se registró la pérdida de peso como $\mathrm{H}_{2} \mathrm{O}$. 
Tabla 1. Requisitos físico-químicos para la panela.

\begin{tabular}{|c|c|c|c|c|}
\hline \multirow{3}{*}{ REQUISITO } & \multicolumn{2}{|c|}{ EN BLOQUE } & \multicolumn{2}{|c|}{$\begin{array}{l}\text { GRANULADA O } \\
\text { PULVERIZADA }\end{array}$} \\
\hline & \multicolumn{2}{|c|}{ VALOR } & \multicolumn{2}{|c|}{ VALOR } \\
\hline & Mín. & Máx. & Mín. & Máx. \\
\hline Humedad, fracción en masa en \% & - & 9 & - & 5 \\
\hline Cenizas, fracción en masa en \% & 0.8 & - & 1 & -- \\
\hline $\begin{array}{l}\text { Azúcares totales (sacarosa), } \\
\text { fracción en masa en \% }\end{array}$ & - & 83 & - & 93 \\
\hline $\begin{array}{l}\text { Azúcares reductores (glucosa), } \\
\text { fracción en masa en\% }\end{array}$ & 5.5 & - & 5 & - \\
\hline Proteínas, en \% ( $\mathrm{N} \times 6.25)$ & $0.20 \%$ & - & $0.20 \%$ & - \\
\hline Potasio en mg/10o g & 100 & -- & 100 & - \\
\hline Calcio en $\mathrm{mg} / 100 \mathrm{~g}$ & 10 & -- & 10 & - \\
\hline Fósforo en mg/10o g & 5 & -- & 5 & - \\
\hline Hierro en $\mathrm{mg} / 100 \mathrm{~g}$ & 1.5 & -- & 1.5 & - \\
\hline Colorantes & Ausencia & & Ausencia & \\
\hline
\end{tabular}

Fuente: Icontec (2009).

$$
\% H=\frac{((W c+W m)-W f)}{W m} \times 100
$$

Donde: $\mathrm{H}$ es la humedad (\%), Wc es el peso de la caja vacía (g), Wm es el peso de muestra (g), y Wf es el peso final de la caja más muestra (g).

\section{Azúcares totales}

Existen diversos métodos de cuantificación de carbohidratos basados en la capacidad reductora de los azúcares que tienen libre el grupo carbonilo. Estos carbohidratos son capaces de reducir elementos como, por ejemplo, el cobre $\left(\mathrm{Cu}^{+2}\right)$, el hierro $\left(\mathrm{Fe}^{+3}\right)$, o el yodo $\left(\mathrm{I}^{\circ}\right)$. En el caso específico del cobre, este se reduce desde $\mathrm{Cu}^{+2} \mathrm{a} \mathrm{Cu}^{+1}$. En este sentido, en el método de Lane y Eynon (1934), se hace reaccionar sulfato cúprico con azúcar reductor en medio alcalino, de modo que se forma óxido cuproso, el cual forma un precipitado rojo ladrillo. Para este caso, la determinación de azúcares se realizó con base en la AOAC 923.09 (AOAC, 2005), determinación de azúcares invertidos por el método volumétrico general de Lane Eynon.

Se utilizó sacarosa grado reactivo (PanReac AppliChem, España, lote 0000460966); tartrato de sodio potasio pureza 99.5\% (Carlo Erba, Italia, lote V4G965104I); sulfato de cobre pentahidratado pureza 99.4\% (Merck, EE.UU., lote AMo686590); hidróxido de sodio pureza 99.4\% (Merck, EE.UU., lote B1360898); azul de metileno pureza 94.3\% (PanReac AppliChem, España, lote 0000797846); ácido clorhídrico concentrado pureza $37.4 \%$ (Merck, EE.UU., lote K48322117).

Se pesó, en una balanza analítica Ohaus, aproximadamente $1 \mathrm{~g}$ de muestra homogeneizada; debido al alto contenido de azúcares, se disolvió en $50 \mathrm{~mL}$ de agua desionizada y se le agregó $1 \mathrm{~mL}$ de ácido clorhídrico concentrado. La solución se sometió a ebullición por 3 min. De forma paralela se realizó el título de sacarosa, en el cual se pesaron 0.5 g de sacarosa, se disolvieron en $25 \mathrm{~mL}$ de agua y se le agregaron $0.5 \mathrm{~mL}$ de ácido clorhídrico concentrado; también se sometió a ebullición por 3 min. Esto se realizó a fin de acelerar el proceso de hidrólisis de la sacarosa, con el propósito de formar azúcares reductores como, por ejemplo, glucosa y fructosa. Se dejó enfriar y se llevó a un pH de 7-9 con hidróxido de sodio 40\%. Luego, las muestras se llevaron a un volumen de $100 \mathrm{~mL}$ en un matraz aforado y la sacarosa a un matraz aforado de $50 \mathrm{~mL}$. En un beaker de $600 \mathrm{~mL}$ se adicionaron $5 \mathrm{~mL}$ de solución Fehling $\mathrm{A}$ alcalina de tartrato de sodio potasio y $5 \mathrm{~mL}$ de solución Fehling B de sulfato de cobre, se le agregaron perlas de ebullición y se sometió a calentamiento. La muestra se introdujo en una bureta de $25 \mathrm{~mL}$, luego a la solución Fehling en ebullición se le agregaron $5 \mathrm{~mL}$ de muestra y en el cambio de color de la solución a rojo ladrillo se le adicionó $1 \mathrm{~mL}$ de indicador azul de metileno. Se continuó con la titulación en la plancha de calentamiento hasta que la solución viró de azul púrpura a rojo ladrillo. Se realizó el mismo proceso para la sacarosa. El volumen de titulación gastado en la muestra se utilizó para los cálculos correspondientes junto con el volumen de titulación de la sacarosa. Este método utiliza azul de metileno como indicador, el cual es decolorado una vez que todo el cobre ha sido reducido, lo que indica el fin de la titulación.

\section{Determinación del título de sacarosa}

$$
T s=\frac{W s \times V t}{D}
$$

Donde: Ts es el título de sacarosa, Ws es el peso de sacarosa (g), Vt es el volumen de titulación $(\mathrm{mL})$ y $\mathrm{D}$ es la dilución $(\mathrm{mL})$.

$$
\% A T=\frac{T s \times D}{W m \times V t} \times 100
$$

Donde: \%AT son los azúcares totales expresados como sacarosa ( $\mathrm{g} / 100 \mathrm{~g})$, Ts es el título de sacarosa, Wm es el peso de muestra (g), Vt es el volumen de titulación $(\mathrm{mL})$, y D es la dilución $(\mathrm{mL})$.

\section{Parámetros termodinámicos \\ Coeficiente de actividad}

La ley de Raoult puede utilizarse en el agua líquida de un alimento con el fin de calcular, de manera independiente, la actividad acuosa. Esto si se asume que la fase de vapor se comporta como un gas ideal y las tendencias de escape de los líquidos son independientes de la presión. A presión y temperatura constantes, la actividad acuosa de una sustancia es igual a la fracción molar de agua en la solución. Cuando existen desviaciones de la idealidad se utiliza un coeficiente de actividad $(\gamma)$, en cuyo caso y para el caso de un sistema binario la ecuación que corresponde a la ley de Raoult se escribe de la forma siguiente (Alvarado, 2012; Romo y Criollo, 1977):

$$
a_{W}=(\gamma) X_{W}
$$


Donde: $a_{w}$ es la actividad acuosa, $\gamma$ es el coeficiente de actividad, y $\mathrm{X}_{\mathrm{w}}$ es la fracción molar del agua.

La fracción molar de agua se determinó por medio de los valores de humedad y azúcares totales expresados como sacarosa de la siguiente manera:

$$
\begin{aligned}
& X_{W}=\frac{n_{\mathrm{H}_{2} \mathrm{O}}}{n_{\mathrm{H}_{2} \mathrm{O}}+n_{\mathrm{AT}}} \\
& =\frac{\% \mathrm{H} / p m \mathrm{H}_{2} \mathrm{O}}{\% \mathrm{H} / p m \mathrm{H}_{2} \mathrm{O}+\% A T / p m C_{12} \mathrm{H}_{22} \mathrm{O}_{11}}
\end{aligned}
$$

Donde: \%H: Humedad (g/10og), \%AT son azúcares totales como sacarosa ( $\mathrm{g} / 100 \mathrm{~g}), \mathrm{pm}_{2} \mathrm{O}$ es el peso molecular del agua $(18.01528 \mathrm{~g} / \mathrm{mol})$, y $\mathrm{pm} \mathrm{C}_{12} \mathrm{H}_{22} \mathrm{O}_{11}$ es el peso molecular de la sacarosa $(342.2965 \mathrm{~g} / \mathrm{mol})$.

\section{Variación de la entalpía molar parcial}

La variación de la entalpía molar parcial se obtuvo como la pendiente de la ecuación lineal de la relación entre el coeficiente de actividad y el inverso de la temperatura absoluta.

\section{Análisis estadístico}

Los cálculos de medidas de tendencia central, desviación y gráficas de correlación se realizaron en Excel (Microsoft Office 2016). Se obtuvieron las curvas del comportamiento de la actividad de agua de muestras de panela con relación al tiempo de almacenamiento, al contenido de humedad y al contenido de azúcares totales; también, del comportamiento de la humedad y los azúcares totales con relación al tiempo de almacenamiento. Adicionalmente, se obtuvieron gráficas del comportamiento de cada tipo de panela a través del tiempo de almacenamiento y se establecieron relaciones entre el coeficiente de actividad y el inverso de la temperatura absoluta, y entre el In (actividad acuosa/tiempo) y el inverso de la temperatura absoluta.

\section{RESULTADOS Y DISCUSIÓN}

\section{Parámetros fisicoquímicos}

\section{Actividad de agua}

En la (Fig. 1) se observa el comportamiento de la actividad de agua de muestras comerciales de panela (PR, PC y PG) durante 129 días de almacenamiento a dos temperaturas (ambiente: $30 \pm 2{ }^{\circ} \mathrm{C}$; acelerada: $40 \pm 2{ }^{\circ} \mathrm{C}$ ).

La actividad acuosa $\left(a_{w}\right)$ se define como la relación entre la presión de vapor de agua del alimento y la presión de vapor del agua pura a la misma temperatura; a su vez, la actividad acuosa de un producto se relaciona con la humedad relativa en equilibrio, en un medio cerrado (Barbosa-Cánovas et al.,
2007). También es la humedad en equilibrio de un producto, determinada por la presión parcial del vapor de agua en su superficie (Vanaclocha y Requena, 2003). El valor $a_{w}$ depende de la composición, la temperatura y el contenido en agua del producto. Estudiar la actividad acuosa es de gran importancia para desarrollar y mejorar la estabilidad alimentaria, ya que el papel del agua se considera crítico en términos de calidad microbiana, química y sensorial (Barbosa-Cánovas et al., 2007).

Al observar la (Fig. 1) se aprecia un incremento en la actividad acuosa con relación al tiempo de almacenamiento para el caso de la presentación PG, en sus dos ambientes de almacenamiento. En las otras presentaciones, PR y PC, no fue posible apreciar un comportamiento claro o proporcional con el tiempo de almacenamiento, como consecuencia de una posible heterogeneidad entre las muestras a nivel de lote, así como de las propiedades plásticas del producto que dificultaron su homogeneización y la obtención de una porción de ensayo representativa, en especial para tiempos de almacenamiento prolongados. Al momento del cuarteo del producto para análisis se observaron diferencias de textura entre las caras expuestas y su interior. Dichas diferencias pueden explicarse por el fenómeno de migración de agua en el producto, ya que dependiendo del proceso de sorción o desorción de agua, la actividad acuosa en la superficie de un alimento puede ser muy diferente a la de su interior. Para el caso de alimentos con altos contenidos de agua, al entrar en contacto con aire seco se produce la desorción de agua y, por tanto, se obtiene una disminución de $\mathrm{a}_{\mathrm{w}}$ en la interfaz que promueve a su vez el transporte de solutos hacia la interfaz que acompaña la migración del agua. En el caso de alimentos con bajo contenido de agua, como, por ejemplo, la panela, al entrar en contacto con aire húmedo la actividad acuosa de la interfaz es mayor a la del interior del producto y los fenómenos difusivos del agua se dan desde la superficie hacia el interior del sólido (Barbosa-Cánovas et al., 2007). Lo anterior se vio reflejado en los cambios de textura y de color en las presentaciones PR y PC, ya que se tornaron blandas y para el caso de PG se observó aglomeración, lo cual limita la posterior solubilidad de los azúcares (Alvarado, 2012). 


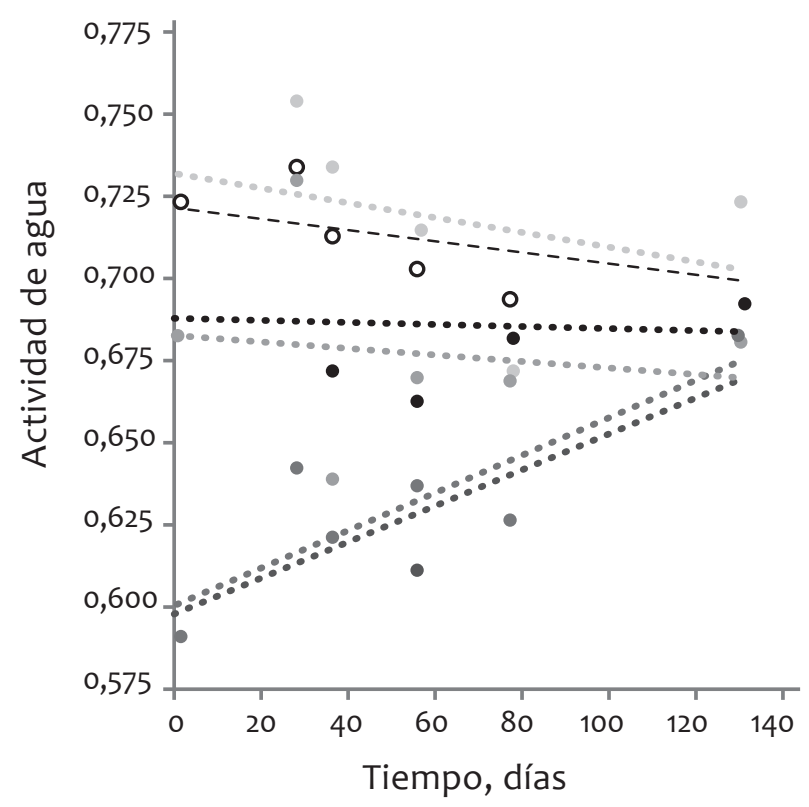

- PR-Amb PC-Amb - PG-Amb PR-Ace PC-Ace

Figura 1. Comportamiento de la actividad de agua de muestras de panela con relación al tiempo de almacenamiento. PR: panela redonda; PC: panela cuadrada; PG: panela granulada; Amb: almacenamiento a temperatura de $30 \pm 2{ }^{\circ} \mathrm{C}$; Ace: almacenamiento a temperatura de $40 \pm 2{ }^{\circ} \mathrm{C}$.

En la (Fig. 2) se observa el comportamiento de la actividad de agua de muestras comerciales de panela (PR, PC y PG) a dos temperaturas de almacenamiento (ambiente: $30 \pm 2{ }^{\circ} \mathrm{C}$; acelerada: $40 \pm 2{ }^{\circ} \mathrm{C}$ ), con relación a la humedad de las muestras.

Se observa en la (Fig. 2) para las presentaciones PG y PC una relación proporcional entre la actividad acuosa y la humedad, es decir, a mayor humedad se da un incremento del valor de $a_{w}$; para la PR no fue notable este comportamiento pues no muestra una tendencia uniforme, lo cual es probable que se debiera a que el muestreo no fue representativo, pues pudo venir sectorizado, por lo que al no tener un mismo modelo de muestreo en todo el estudio no permitió evidenciar un comportamiento como tal. Estudiar estas variables permite comprender la evolución de la calidad del producto. De los resultados obtenidos se observa que se debe controlar la humedad en la conservación del producto, ya que a valores entre 0.7 y 0.8 de $a_{w}$ se alcanza la máxima velocidad del pardeamiento no enzimático o reacciones enzimáticas que provocan cambios en atributos sensoriales (Badui Dergal, 2006). En la (Fig. 3) se observa el comportamiento de la actividad de agua de muestras comerciales de panela (PR, PC y PG) a dos temperaturas de almacenamiento (ambiente: $30 \pm 2$ ${ }^{\circ} \mathrm{C}$; acelerada: $40 \pm 2{ }^{\circ} \mathrm{C}$ ), con relación al porcentaje de azúcares totales, como, por ejemplo, sacarosa.

Se puede observar en la (Fig. 3) que para la presentación PG en almacenamiento acelerado y ambiente existe una rela- ción directamente proporcional con la $\mathrm{a}_{\mathrm{w}}$. En las presentaciones PR y PC es notorio que, a mayor $a_{w}$, menor contenido de azúcares como sacarosa. Esto puede ser producto de la inversión de sacarosa, ya que un aumento de la actividad de acuosa conlleva a una mayor velocidad de reacción de la inversión de azúcares, debido a una mayor movilidad de los reactivos que incrementa el contenido de azúcares reductores como, por ejemplo, glucosa y fructosa, lo que genera un oscurecimiento en las presentaciones (Mosquera et al., 2007).

La mejor forma de medir la disponibilidad de agua es mediante la $a_{w}$, y en un alimento se puede reducir al aumentar la concentración de solutos en la fase acuosa de los alimentos mediante la extracción del agua o mediante la adición de solutos. La $a_{w}$ es uno de los factores intrínsecos que posibilitan o dificultan el crecimiento microbiano en los alimentos. Por esto, la medición de esta es importante para controlar dicho crecimiento. Tiene incidencia sobre las características de calidad, tales como textura, sabor, color, gusto, valor nutricional del producto y su tiempo de conservación. Los microorganismos necesitan la presencia de agua, en una forma disponible, a fin de crecer y llevar a cabo sus funciones metabólicas. La disminución de la $a_{w}$ frena el crecimiento de los microorganismos, las reacciones catalizadas por enzimas (principalmente hidrolasas) y, posteriormente, el pardeamiento no enzimático. Sin embargo, la oxidación de los lípidos es la excepción, ya que la velocidad se incrementa en el alimento seco (Mejía Cárdenas, 2003).

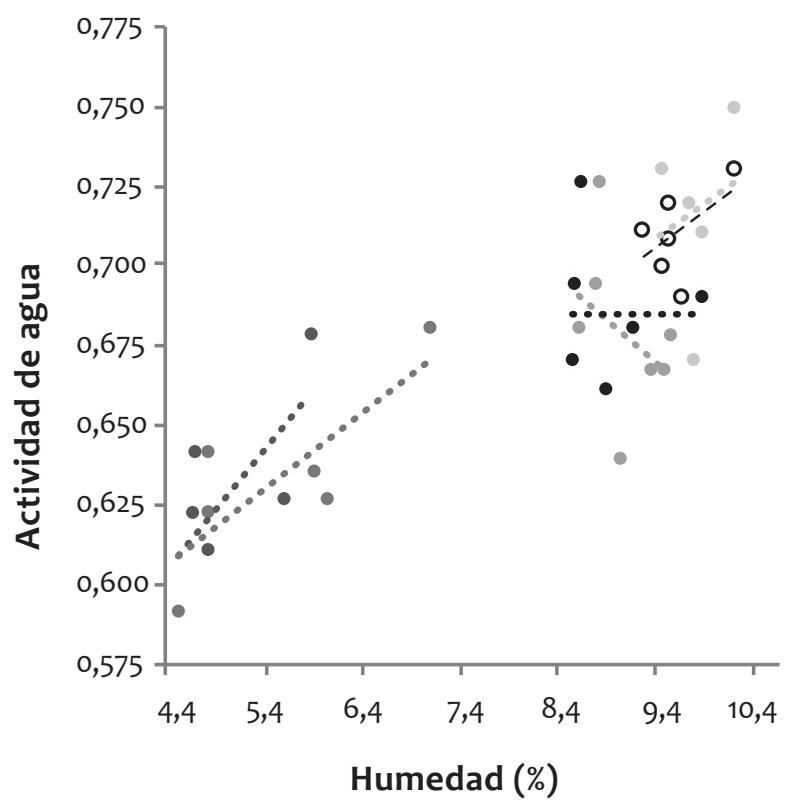

- PR-Amb •PC-Amb •PG-Amb -PR-Ace OPC-Ace •PG-Ace

Figura 2. Comportamiento de la actividad de agua de muestras de panela con relación al contenido de humedad. PR: panela redonda; PC: panela cuadrada; PG: panela granulada; Amb: almacenamiento a temperatura de $30 \pm 2{ }^{\circ} \mathrm{C}$; Ace: almacenamiento a temperatura de $40 \pm 2{ }^{\circ} \mathrm{C}$. 


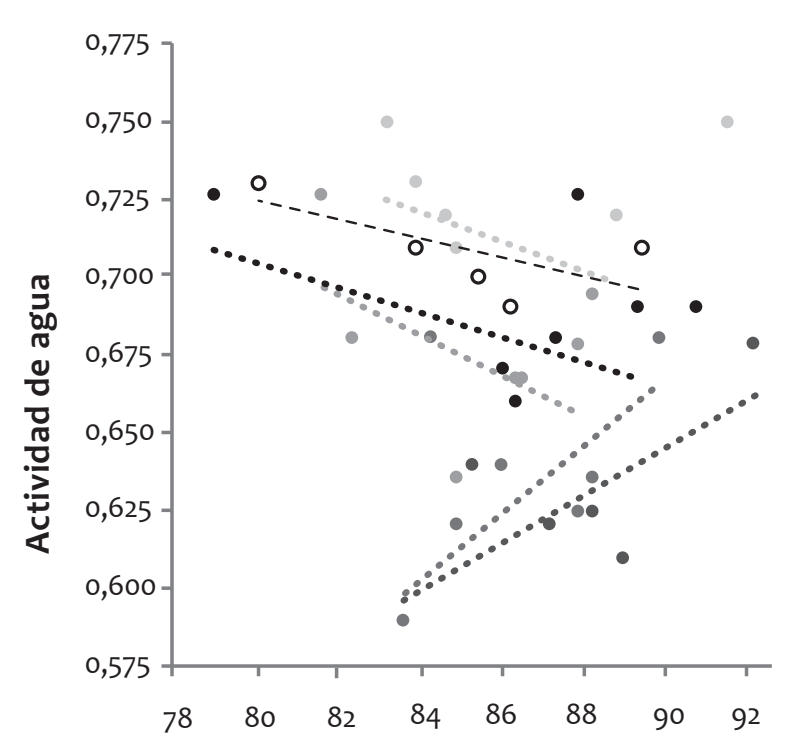

Azúcares totales como sacarosa (\%)

- PR-Amb - PC-Amb - PG-Amb -PR-Ace O PC-Ace •PG-Ace

Figura 3. Comportamiento de la actividad de agua de muestras de panela con relación al contenido de azúcares totales expresados como sacarosa. PR: panela redonda; pc: panela cuadrada; pg: panela granulada; Amb: almacenamiento a temperatura de $30 \pm 2{ }^{\circ} \mathrm{C}$; Ace: almacenamiento a temperatura de $40 \pm 2{ }^{\circ} \mathrm{C}$.

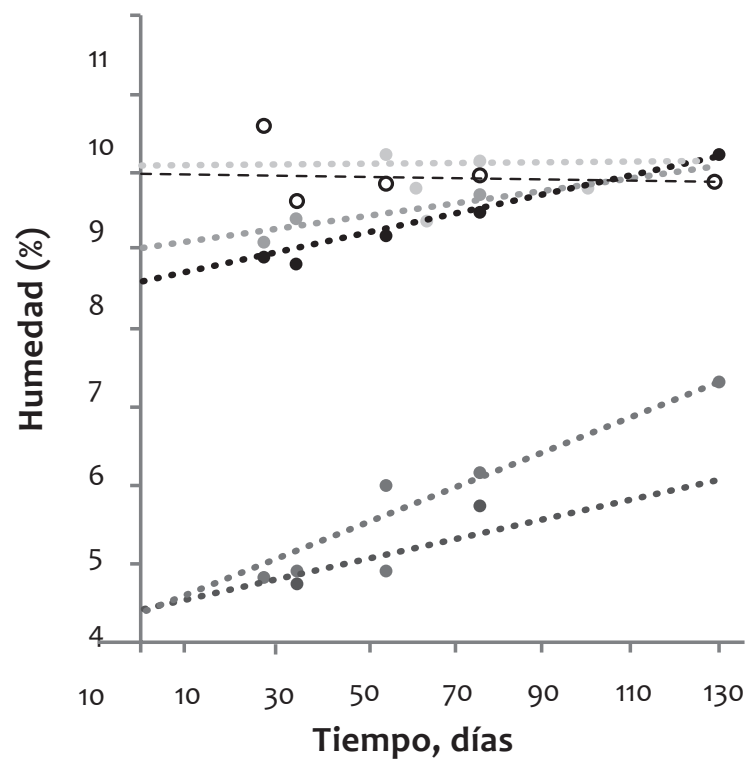

- PR-Amb - PC-Amb - PG-Amb - PR-Ace O PC-Ace •PG-Ace

Figura 4. Comportamiento de la humedad (\%) de muestras de panela con relación al tiempo de almacenamiento. PR: panela redonda; pc: panela cuadrada; pg: panela granulada; Amb: almacenamiento a temperatura de $30 \pm 2{ }^{\circ} \mathrm{C}$; Ace: almacenamiento a temperatura de $40 \pm 2{ }^{\circ} \mathrm{C}$.

\section{Humedad}

En la (Fig. 4) se observa el comportamiento de la humedad de muestras comerciales de panela (PR, PC y PG) durante 129 días de almacenamiento a dos temperaturas (ambiente: $30 \pm 2$ ${ }^{\circ} \mathrm{C}$; acelerada: $40 \pm 2{ }^{\circ} \mathrm{C}$ ).

La determinación del contenido de humedad de los alimentos es una propiedad importante y ampliamente usada en el procesamiento y el control de calidad, ya que indica la cantidad de agua involucrada en su composición. La medida de la humedad en procesos de deshidratación consiste en la determinación de la pérdida de masa que sufre un alimento cuando se somete a una combinación tiempo-temperatura adecuada. El residuo que se obtiene se conoce como "sólidos totales" o "materia seca".

En los tejidos vegetales y animales existen dos formas generales: agua libre y agua ligada, como soluto o como solvente; en forma libre, al formar hidratos o como agua adsorbida. En algunos alimentos solo una parte del agua que contienen se pierde a esta temperatura. El resto (agua combinada o adsorbida) es difícil de eliminar y parece estar asociada a las proteínas presentes. La proporción de agua libre perdida aumenta al elevar la temperatura, por lo que es importante comparar únicamente los resultados obtenidos cuando se usan las mismas condiciones de secado (García Martínez y Fernández Segovia, 2012).

Según la Resolución 779 de 2006 (MinSalud, 2006), como criterio máximo de humedad se establece el $9 \%$ para panela en bloque y el $5 \%$ para granulada. Las muestras que se utilizaron en el estudio en el tiempo ø cumplieron con la especificación de la resolución. A partir del tiempo 1 de almacenamiento las presentaciones PR y PC presentaron un desvío en el cumplimiento de la especificación para las dos condiciones de almacenamiento; esto pudo deberse a defectos en el empaque, lo cual permitió que la humedad aumentara. Al final del estudio las tres presentaciones dejaron de cumplir con la resolución, y arrojaron que en condición acelerada $\left(40 \pm 2{ }^{\circ} \mathrm{C}\right)$ se obtenía mayor humedad respecto a la condición de almacenamiento a ambiente $\left(30 \pm 2{ }^{\circ} \mathrm{C}\right)$; esto puede deberse a que si la humedad del producto es menor que el porcentaje de humedad relativa (\%HR) de almacenamiento, el agua ingresará al empaque del producto, de manera que aumenta la humedad por el fenómeno de difusión, ya que esta depende, en gran medida, del logro del equilibrio termodinámico, porque la pérdida o ganancia de humedad de una región o componente alimenticio a otra región o componente ocurrirá continuamente para alcanzar el equilibrio termodinámico con los componentes alimentarios circundantes y ambiente (Barbosa-Cánovas et al., 2007).

En los alimentos se puede controlar mediante la difusión molecular el trasporte de agua en sólidos, en el que la fuerza motriz puede ser un gradiente de concentración o gradiente de contenido de humedad. La ley de difusión de Fick se refiere al movimiento de un componente a través de una mezcla 
binaria bajo un gradiente constante de presión de vapor a temperatura constante. La fuerza impulsora es, por tanto, el potencial químico o la diferencia de presión de vapor entre las dos regiones (Barbosa-Cánovas et al., 2007).

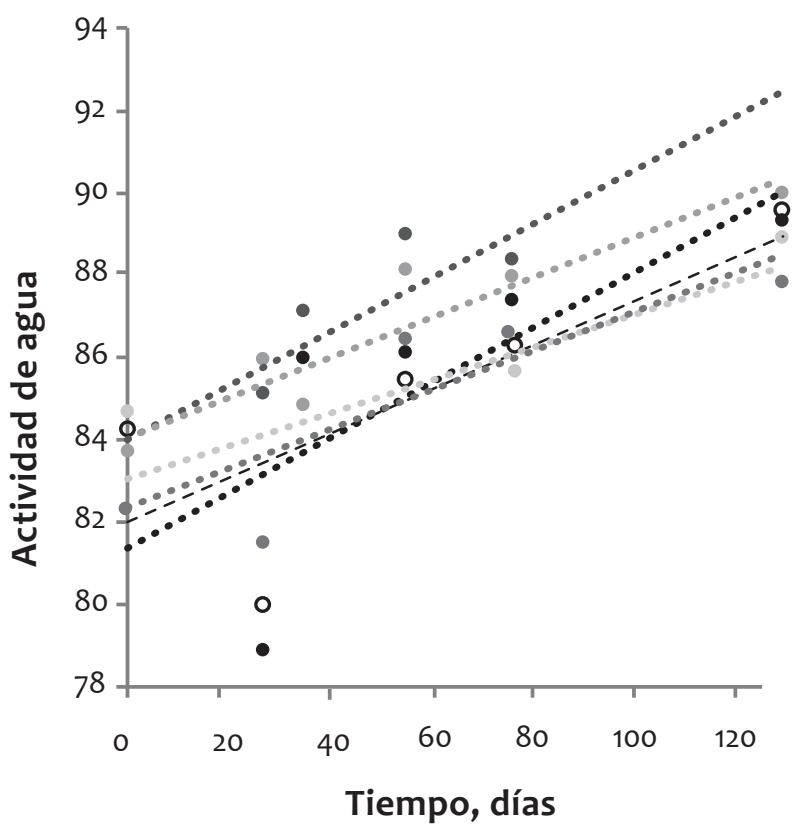

- PR-Amb - PC-Amb •PG-Amb - PR-Ace o PC-Ace •PG-Ace

Figura 5. Comportamiento de los azúcares totales como sacarosa (\%) de muestras de panela con relación al tiempo de almacenamiento. PR: panela redonda; PC: panela cuadrada; PG: panela granulada; Amb: almacenamiento a temperatura de $30 \pm 2{ }^{\circ} \mathrm{C}$; Ace: almacenamiento a temperatura de $40 \pm 2^{\circ} \mathrm{C}$.

\section{Azúcares totales}

En la (Fig. 5) se observa el comportamiento del porcentaje de azúcares totales, como, por ejemplo, sacarosa de muestras comerciales de panela ( $P R, P C$ y $P G)$, durante 129 días de almacenamiento a dos temperaturas (ambiente: $30 \pm 2{ }^{\circ} \mathrm{C}$; acelerada: $40 \pm 2{ }^{\circ} \mathrm{C}$ ).

Se observa una relación directamente proporcional entre el contenido de azúcares totales y el tiempo de almacenamiento para las tres presentaciones en sus dos condiciones de almacenamiento, por lo que se infiere un error sistemático al momento de realizar los análisis de azúcares totales por el método Lane Eynon, pues según la teoría se espera que el contenido de sacarosa disminuya debido al proceso de inversión de esta en azúcares reductores (lo cual se ve reflejado en la apariencia del producto); por tanto, el contenido de azúcares totales se mantendría constante. Otra razón pudo ser que, a pesar de que las muestras eran del mismo lote, este pudo venir sectorizado debido a los procesos de batido o moldeo en la elaboración de la panela, lo cual, al momento de hacer los análisis, afectó en el proceso de homogeneización, pues el cuarteo no fue representativo ya que no se homogenizo toda la muestra. Controlar las variables del método de azúcares totales (entre ellas la temperatura de calentamiento al momento de realizar la titulación) permite garantizar una mayor precisión del método analítico, de modo que sea posible evidenciar pequeños cambios en el parámetro medido, así como diferenciar del error aleatorio del método, lo que incluya la selección de la porción de muestra para el análisis, como, por ejemplo, la profundidad muestreada de la superficie expuesta o de los puntos del interior del producto. También el método de Lane Eynon presenta un inconveniente en la determinación de azúcares, ya que la panela puede contener otras especies que pueden generar interferencias, entre estas, otros azúcares como las cetosas. Estas pueden sufrir efectos de tautomerización por la alcalinidad del medio, así como las especies inorgánicas y las moléculas con grupos aldehído en su estructura susceptibles a oxidación que afectan el resultado del contenido real de azúcares en la panela (Zambrano Herrera, 2011). Dado que en los productos derivados de la caña de azúcar — como es el caso de la panela- se podría presentar contenido de furfural y derivados que, al ser aldehídos, actúan como agentes reductores (Instituto Cubano de Investigaciones de los Derivados de la Caña de Azúcar-Icidca, 1986), es decir, en el momento del análisis reducen el cobre y se suman como un interferente, se recomienda el análisis de estos azúcares por cromatografía líquida de alta resolución HPLC, ya que se obtienen resultados con mayor exactitud debido a que es específico y selectivo para estos azúcares, aunque en comparación con la otra técnica suele ser más costosa (Zambrano Herrera, 2011). A los 27 días de almacenamiento se observa en las presentaciones PR y PC (Fig. 5), en ambiente y acelerado, una disminución notable del contenido de azúcares que se pudo deber al incremento de la actividad acuosa con relación a los demás tiempos.

En la (Fig. 6) se aprecian los cambios en las características físicas, tales como el color y la textura, en las muestras de panela analizadas.

Se aprecia cómo en el inicio del estudio las panelas redonda y cuadrada tenían apariencia de sólido duro quebradizo, y al final se tornaron en un sólido plástico o blando de color más intenso. La panela granulada, por su parte, era un polvo granulado suelto, pero al final se aglutinó y adquirió también un color más oscuro; dicha textura puede estar relacionada con el incremento en la humedad y la actividad acuosa. Esto puede deberse a que los reactivos se disuelven y movilizan en la panela, de forma que aumentan la velocidad de la reacción de Maillard, responsable de los aromas, los sabores y el color dorado deseables e indeseables en los alimentos. Esta reacción implica una amina no protonada que actúa como un nucleófilo al atacar un grupo carbonilo, en el cual los azúcares reductores son las fuentes más comunes de grupo carbonilo (Barbosa-Cánovas et al., 2007), por lo que se presume un incremento en los azúcares reductores. 


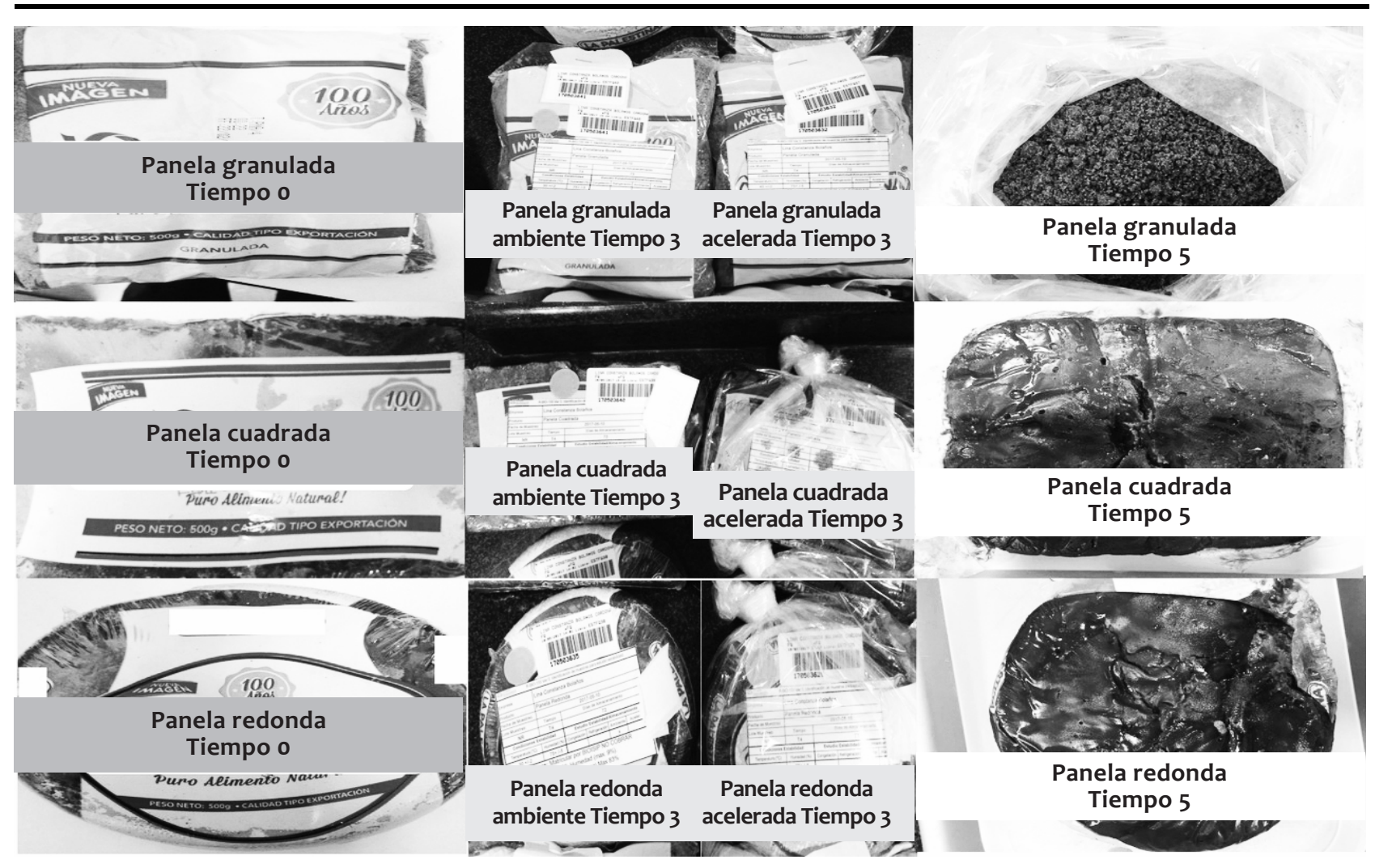

Figura 6. Muestras de panela a través del tiempo de almacenamiento.

\section{Parámetros termodinámicos \\ Coeficiente de actividad}

Con los resultados obtenidos de los parámetros de $\% \mathrm{H}, \mathrm{a}_{\mathrm{w}}$ y azúcares totales, se determinó el coeficiente de actividad por medio de la ley de Raoult (Barbosa-Cánovas et al., 2007). En la (Tabla 2) se presentan los valores de coeficientes de actividad de muestras de panelas para dos temperaturas de almacenamiento.

Tabla 2. Coeficiente de actividad de muestras de panelas para dos temperaturas de almacenamiento.

\begin{tabular}{cccc}
\hline \multirow{2}{*}{ T, K } & \multicolumn{3}{c}{$\Gamma$} \\
\cline { 2 - 4 } & PR & PC & PG \\
303.15 & $1.027 \pm 0.027$ & $1.048 \pm 0.036$ & $1.206 \pm 0.043$ \\
313.15 & $1.008 \pm 0.043$ & $1.042 \pm 0.017$ & $1.164 \pm 0.050$
\end{tabular}

PR: panela redonda; PC: panela cuadrada; PG: panela granulada.

El $\gamma$ cambia con la temperatura, pues a mayor temperatura de almacenamiento disminuye su valor, lo que indica menores desviaciones del comportamiento como un gas ideal conforme se incrementa la temperatura a las que se encuentran las muestras de panela.

El $\gamma$ permite conocer la desviación del comportamiento ideal de un compuesto, pues a valores de coeficiente de actividad igual a 1 el comportamiento sería de un gas ideal, al ser un valor diferente indica desviaciones del comportamiento ideal, lo cual se debe a diferentes causas relacionadas con propiedades moleculares específicas de la especie en la solución. Entre estas se encuetran el tamaño de solutos, las fuerzas intermoleculares y los efectos de solvatación que aumentan con la concentración (Barbosa-Cánovas et al., 2007). Para el caso de las PC y PR fueron valores iguales a uno, pero en la presentación PG el valor fue mayor que uno, ya que en esta el contenido de sólidos es mayor si se compara con el del agua que es mínimo, lo que genera desviaciones del comportamiento ideal. Esta desviación puede atribuirse al elevado número de moléculas de sacarosa con respecto a las de agua, ya que, según la teoría, estas partículas reducen la tendencia al escape o fugacidad del agua (Labuza y Altunakar, 2008). Por tanto, a medida que aumenta la concentración, el coeficiente de actividad disminuye, de manera que deprime $a_{w}$ de forma significativa. 


\section{Variación de la entalpía molar parcial}

Tabla 3. Entalpía molar parcial en las muestras de panela.

\begin{tabular}{cccc}
\hline & \multicolumn{3}{c}{ LN(г) } \\
\cline { 2 - 4 } 1/T (1/K) & PR & PC & PG \\
$3.299 . \mathrm{E}-03$ & 0.027 & 0.047 & 0.187 \\
$3.193 . \mathrm{E}-03$ & 0.008 & 0.041 & 0.152 \\
Pendiente & 179.40 & 54.95 & 335.91 \\
& 1491.57 & 456.85 & 2792.73
\end{tabular}

PR: panela redonda; PC : panela cuadrada; PG: panela granulada.

La variación de la entalpía molar parcial la determina el medio de la pendiente de la relación entre el logaritmo natural del coeficiente de actividad comparada con el inverso de la temperatura de almacenamiento para las tres presentaciones (Tabla 3), donde se obtiene para la panela redonda 1491.57 $\mathrm{J} / \mathrm{g} \cdot \mathrm{mol}$, para panela cuadrada $456.85 \mathrm{~J} / \mathrm{g} \cdot \mathrm{mol}$, y para panela granulada $2792.73 \mathrm{~J} / \mathrm{g} \cdot \mathrm{mol}$.

\section{Parámetros cinéticos}

A fin de predecir el tiempo de vida útil se aplicaron cinéticas de deterioro (Tabla 4). Se evaluaron diferentes órdenes de reacción con el propósito de determinar con cuál se observaban mejores resultados y mayor ajuste en la relación de $a_{w}$ respecto al tiempo de almacenamiento. Entre los procesos de deterioro de los alimentos que se rigen por la ecuación de orden cero se encuentra la degradación enzimática, el pardeamiento no enzimático y la oxidación de lípidos que lleva a la producción de olores rancios; para la ecuación de primer orden están las pérdidas de vitaminas y proteínas, la muerte o el desarrollo microbiano, la pérdida de color por oxidación y la pérdida de textura en tratamientos térmicos (Vanaclocha y Requena, 2003).

En la (Tabla 5) se presentan los parámetros cinéticos de muestras de panelas para dos temperaturas de almacenamiento.

Tabla 4. Expresiones para los tiempos de vida útil y media de acuerdo con el orden de reacción.

\begin{tabular}{ccc}
\hline $\begin{array}{c}\text { ORDEN APARENTE } \\
\text { DE LA REACCIÓN }\end{array}$ & $\begin{array}{c}\text { ECUACIÓN TIEMPO DE } \\
\text { VIDA ÚTIL (TU) }\end{array}$ & $\begin{array}{c}\text { ECUACIÓN TIEMPO DE } \\
\text { VIDA MEDIA (T1/2) }\end{array}$ \\
$Q_{f}=Q_{0}-k t_{u}$ & $t_{1 / 2}=Q_{0} / 2 k$ \\
1 & $\ln Q_{f}=\ln Q_{0}-k t_{u}$ & $t_{1 / 2}=\ln 2 / k$ \\
2 & $1 / Q_{f}=1 / Q_{0}+k t_{u}$ & $t_{1 / 2}=1 / k Q_{0}$
\end{tabular}

Fuente: elaboración propia.

Tabla 5. Parámetros cinéticos de muestras de panelas para dos temperaturas de almacenamiento.

\begin{tabular}{|c|c|c|c|c|c|c|c|}
\hline ORDEN & PARÁMETROS & PR-AMB & PR-ACE & РC-АMB & PC-ACE & PG-AMB & PG-ACE \\
\hline & $\mathrm{Q}_{0}$ & 6.87E-01 & $6.83 \mathrm{E}-01$ & 7.28E-01 & $7.18 \mathrm{E}-01$ & $5.98 \mathrm{E}-01$ & 6.01E-01 \\
\hline \multirow[t]{3}{*}{0} & $\mathrm{~K}$ & $-4.15 E-05$ & $-1.03 E-04$ & $-2.13 E-04$ & $-1.62 \mathrm{E}-04$ & 5.49E-04 & 5.64E-04 \\
\hline & $\mathrm{R}^{2}$ & 0.0059 & 0.0247 & 0.1300 & 0.2664 & 0.6785 & 0.7477 \\
\hline & $\ln Q_{0}$ & $-3.76 \mathrm{E}-01$ & $-3.83 E-01$ & $-3.18 \mathrm{E}-01$ & $-3.31 \mathrm{E}-01$ & $-5.14 \mathrm{E}-01$ & $-5.08 \mathrm{E}-01$ \\
\hline \multirow[t]{3}{*}{1} & $\mathrm{~K}$ & $-5.49 E-05$ & $-1.42 \mathrm{E}-04$ & $-2.99 E-04$ & $-2.28 \mathrm{E}-04$ & $8.62 \mathrm{E}-04$ & 8.85E-04 \\
\hline & $\mathrm{R}^{2}$ & 0.0050 & 0.0221 & 0.1281 & 0.2649 & 0.6744 & 0.7417 \\
\hline & $1 / Q_{0}$ & $1.46 \mathrm{E}+00$ & $1.47 \mathrm{E}+00$ & $1.37 \mathrm{E}+00$ & $1.39 \mathrm{E}+00$ & $1.67 \mathrm{E}+00$ & $1.66 \mathrm{E}+00$ \\
\hline \multirow[t]{2}{*}{2} & $\mathrm{~K}$ & $7.22 \mathrm{E}-05$ & $1.94 \mathrm{E}-04$ & $4.20 \mathrm{E}-04$ & $3.21 \mathrm{E}-04$ & $-1.36 \mathrm{E}-03$ & $-1.39 \mathrm{E}-03$ \\
\hline & $\mathrm{R}^{2}$ & 0.0042 & 0.0195 & 0.1261 & 0.2633 & 0.6698 & 0.7347 \\
\hline
\end{tabular}

PR: panela redonda; PC: panela cuadrada; PG: panela granulada; Amb: almacenamiento a temperatura de $30 \pm 2{ }^{\circ} \mathrm{C}$; Ace: almacenamiento a temperatura de $40 \pm 2{ }^{\circ} \mathrm{C}$.

Se observa para la PR y PC que ninguno de los órdenes de reacción mantiene una relación lineal entre la $\mathrm{a}_{w} \mathrm{y}$ el tiempo de almacenamiento en razón a los valores tan bajos de coeficiente de regresión $\mathrm{R}^{2}$. Esto porque no se observó muy bien el comportamiento, pues a pesar de que las muestras eran del mismo lote este pudo venir sectorizado debido a los procesos de batido o de moldeo en la elaboración de la panela que, al momento de hacer los análisis, afectó en el proceso de homogeneización, ya que el cuarteo no fue representativo a causa de que no se homogeneizó toda la muestra. En la presentación PG fue un poco más alto el coeficiente de regresión, ya que no se tuvo ese inconveniente, pues en el proceso de elaboración al final se realiza un batido y, de forma simultánea, la deshidratación de las mieles a cierta temperatura, con el objetivo de obtener los granos esféricos o polvo, por lo que es más representativa. Se determinó el tiempo de vida útil $\left(t_{u}\right)$, con relación a la $a_{w}$, por medio de la ecuación de orden de reacción cero, y se tomó como criterio que en los alimentos una $a_{w}$ inferior a 0.7 se considera el límite inferior, ya que presenta todas las garantías de estabilidad, pues en los alimentos con $a_{w}$ baja (0.61-0.85) las alteraciones microbianas más frecuentes son las producidas por mohos, y 
el valor mínimo $\mathrm{a}_{w}$ de crecimiento para este microorganismo es de 0.70 (Vanaclocha y Requena, 2003).

Los $t_{u}$ obtenidos con relación a la actividad acuosa fueron para panela redonda ambiente de 174 días y acelerada de 29 días; para panela cuadrada ambiente de 132 días y acelerada de 114 días; para panela granulada ambiente de 186 días y acelerada de 175 días. Al sumarle el tiempo de fabricación de las panelas se obtiene, respectivamente: para panela redonda ambiente 196 días, y acelerada 51 días; para panela cuadrada ambiente 154 días, y acelerada 136 días; para panela granulada ambiente 193 días, y acelerada 182 días. El tiempo de vida útil promedio para panela redonda fue de 123 días, para panela cuadrada de 144 días y para panela granulada de 187 días. Es importante observar cómo a medida que se incrementa la temperatura de almacenamiento se reduce el tiempo de vida útil del alimento.

En varias ocasiones la temperatura de almacenamiento del producto es diferente a las temperaturas de almacenamiento en que se trabajó el estudio, por lo que la ecuación de Arhenius permite la determinación del tiempo a la temperatura de interés, ya que esta describe el efecto de la temperatura sobre la velocidad del deterioro del alimento (Vanaclocha y Requena, 2003).

De la pendiente y el intercepto obtenido del logaritmo natural de la velocidad de cambio, definida por la relación $\mathrm{a}_{\mathrm{w}} /$ tiempo versus el inverso de la temperatura absoluta en muestras comerciales de panela (Tabla 6), se pudo estimar el tiempo de vida útil a $25^{\circ} \mathrm{C}$, de modo que los tiempos de vida útil obtenidos fueron mayores comparados con las dos condiciones del estudio: 398 días para panela redonda, 164 días para panela cuadrada, y 206 días para panela granulada; en la panela redonda se obtuvo un tiempo mayor comparado con las otras presentaciones debido a que esta presentó mayor variación entre el tiempo de vida útil en condición ambiente y acelerado, lo que generó una pendiente más alta y, por consiguiente, un tiempo de vida útil a $25^{\circ} \mathrm{C}$ elevado, pues los valores de actividad acuosa para la panela redonda en sus dos condiciones de almacenamiento mantuvieron tendencias diferentes, de modo que no fue apreciable significativamente la variación de actividad acuosa a través del tiempo en comparación con las otras presentaciones.

Tabla 6. Relación entre el In (actividad acuosa/tiempo) y el inverso de la temperatura absoluta en muestras comerciales de panela.

\begin{tabular}{lccc}
\hline 1/T $[1 / K]$ & \multicolumn{3}{c}{$\mathrm{LN}(\mathrm{AW} / \mathrm{T})[1 / \mathrm{DIA}]$} \\
\cline { 2 - 4 } & $\mathrm{PR}$ & $\mathrm{PC}$ & $\mathrm{PG}$ \\
$3.299 . \mathrm{E}-03$ & -5.664 & -5.391 & -5.621 \\
& & -5.267 & -5.560 \\
3.193.E-03 & -4.317 & -1172.428 & -578.887 \\
Intercepto & -12785.796 & -1.523 & -3.712 \\
Pendiente & 36.512 & &
\end{tabular}

PR: panela redonda; PC: panela cuadrada; PG: panela granulada.

\section{CONCLUSIONES |}

Al finalizar el estudio se evidenció que en la PG se obtuvo mejores resultados en comparación con la PR y PC, es decir, fue más notable el comportamiento de las variables de estudio a través del tiempo, lo cual se pudo deber al proceso de elaboración en la panela (batido y moldeo), o al muestreo realizado al homogeneizar las muestras para el análisis.

La calidad de los alimentos se ve reflejada en las propiedades que influyen en la aceptación del producto por el consumidor; para el caso de la panela, el análisis de la actividad acuosa, de la humedad y de los azúcares es fundamental, ya que determina la calidad del producto.

El estudio de la variable actividad acuosa es de vital importancia, ya que esta influye en distintas características de los alimentos como lo son el color, el olor, el sabor y la textura (la cual se ve afectada en la vida útil de muchos productos), pues se puede predecir la seguridad o la estabilidad con respecto al crecimiento microbiano.

La reacción Maillard está relacionada con el incremento del valor de actividad acuosa y la ganancia de humedad, ya que hay una mayor velocidad de reacción en la inversión de azúcares debido a una mayor movilidad de los reactivos, lo cual se ve reflejado en el oscurecimiento de la panela; por tanto, al final del estudio la PR, la PC y la PG evidenciaron un color café más oscuro con mal apariencia.

Para el análisis de azúcares se recomienda realizarlo por la técnica HPLC (la cual es más confiable), ya que los productos derivados de la caña de azúcar podrían tener furfural y derivados, los cuales son un interferente en la metodología de Lane Eynon, ya que este compuesto es un aldehído que actúa también como un agente reductor.

Se recomienda explorar o relacionar el efecto de las sales durante el almacenamiento de la panela, así como el contenido de nitrógeno, a fin de evidenciar la reacción de Maillard.

\section{AGRADECIMIENTOS}

Los autores agradecen a Laboratorios Ángel Bioindustrial por suministrar los equipos y facilitar la realización de los análisis, en especial a la Dra. María Teresa Ángel por su apoyo y especial interés en la realización de este proyecto. Al Qco. Daniel Velandia-Rodríguez por su apoyo y orientación en la realización del proyecto.

\section{CONFLICTO DE INTERESES}

Los autores no tienen conflicto de intereses.

\section{REFERENCIAS}

- Acurio, L. P. (2010). Determinación en los principales indicadores en el tiempo de vida de anaquel en panela granulada de las unidades productivas de Ingapi y el Paraíso con fines de exportacion al mercado norteamericano. Universidad Técnica de Ambato.

- Alvarado J. d. D. (2012). Propiedades termodinámicas relacionadas con el agua constitutiva de alimentos. Ambato: Imprenta Grafitext. 
- Álvarez Londoño, A. F. (2005). Perfil de producto: panela en Estados Unidos. Recuperado de http://www.panelamonitor.org/media/docrepo/document/files/ perfil-de-producto-panela-estados-unidos.pdf.

- AOAC. (2005). Official Methods of Analysis. Gaithersburg: AOAC International.

- AOAC. (2005). Official Methods of Analysis. Gaithersburg: AOAC International.

- Badui Dergal, S. (2006). Agua. En Química de los alimentos (pp. 1-24). México: Pearson Educación.

- Barbosa-Cánovas, G. V., Fontana, J. A. J., Schmidt, S. J. y Labuza, T. P. (2007). Water Activity in Foods Fundamentals and Applications. Ames: Blackwell Publishing IFT Press.

- Casp Vanaclocha, A. y Abril Requena, J. (2003). Proceso de conservación de alimentos. Madrid: Ediciones Mundi Prensa.

- García Martínez, E. y Fernández Segovia, I. (2012). Determinación de la humedad de un alimento por un método gravimétrico indirecto por desecación. Valencia: Riunet.

- Icontec. (2009). Norma Técnica Colombiana NTC 1311. Bogotá: Instituto Colombiano de Normalizacion Icontec.

- Icidca (Instituto Cubano de Investigaciones de los Derivados de la Caña de Azúcar). (1986). La industria de los derivados de la caña de azúcar. La Habana: Científico-Técnico La Habana.

- Labuza, T. P. y Altunakar, L. (2008). Water Activity Prediction and Moisture Sorption Isotherms. Oxford: Blackwell Publishing Ltd.

- Mejía Cárdenas, A. L. (2003). Evaluación del tiempo de vida útil y estabilidad de las propiedades de calidad de grano reventado de amaranto y sus dos productos. Ibarra, Ecuador: Universidad Técnica del Norte, Facultad de Ingeniería en Ciencias Agropecuarias y Ambientales.

- Resolución 779 de 2006 [Ministerio de Salud y la Protección Social]. Por la cual se establece el reglamento técnico sobre los requisitos sanitarios que se deben cumplir en la producción y comercialización de la panela para consumo humano y se dictan otras disposiciones. Marzo 17 de 2006. DO 46223.

- Mosquera Silvio, A., Carrera, J. E. y Villada, H. S. (2007). Variables que afectan la calidad de la panela. Biotecnología en el Sector Agropecuario y Agroindustrial, 5(1), 17-27.

- Sandoval, G., Mora, W. y Tuz, C. (2007). Producción mecánica de panela granulada.

- Uppal, S. K., Thind, K. S. y Gill, R. S. (2005). Relative Performance of Sugarcane Genotypes for Jaggery (gur) Quality and Storage. Sugar Tech, 7(4), 136-40.

- Zambrano Herrera, A. C. (2011). Estudio comparativo de métodos para la determinación de sacarosa y azúcares reductores en miel virgen de caña utilizados en el Ingenio Pichichí S. A. Universidad Tecnológica de Pereira Facultad de Tecnologías, Programa de Tecnología Química. 